\title{
Special Software Used for Implementing Elements of Hydrographical Network into Hydrogeological Model of Latvia
}

\author{
Kaspars Krauklis ${ }^{1}$, Janis Slangens ${ }^{2}$, \\ ${ }^{1-2}$ Riga Technical University
}

\begin{abstract}
Hydrogeological model (HM) of Latvia (LAMO) is used for simulation of groundwater flow regime in its active zone. Surface waters - rivers, lakes and sea have great influence on groundwater flow. Current LAMO3 version contains 27 layers with $610 \times 951 \times 27=15431877$ spatial grid nodes; the plane approximation step of LAMO3 is $\mathbf{5 0 0}$ meters. Rivers, lakes and sea form hydrographical network (HN) of LAMO3: 462 rivers in 42680 nodes and 128 lakes and sea in 104303 nodes are attached to aquifers. Development, modifying and improving of $\mathrm{HN}$ is an iterative process. Adding or excluding of a particular river or lake must be performed in short time. It is impossible to do it without developing special software, which performs automation of creating the HN and including it into the model. This paper describes some algorithms and software, which were used for $\mathrm{HN}$ creation and its inclusion into LAMO3, such as the data interpolation of river and lake geometry, attaching $\mathrm{HN}$ to the model, immersion of river valleys into the HM body, creation of HN data for a model version with a different plane approximation step, joining of model surfaces for data input for Groundwater Vistas (GV) modelling system and software solution overview, which achieved all objectives of $\mathrm{HN}$ creation.
\end{abstract}

Keywords - Automation, hydrographical network, LAMO, modelling, software.

\section{INTRODUCTION}

Problems of HN creation in LAMO3 [1]-[3] are common in HM development. An essential need for automation of this process is based on rapid evolution of IT hardware and software approaches, with growing opportunity of obtaining, processing and using huge amounts of data. Original software solutions of Environment Modelling Centre of Riga Technical University were adapted using the newest software compilers and by increasing data array sizes [4]. Notable amount of initial data were obtained which described geological layers. It was difficult to find faults in these data manually, because the checking was time consuming. In the creation of the LAMO relief surface [5] more than 1 million isolines were checked and the false level values were corrected.

It was impossible to obtain $\mathrm{HN}$ data for the regional model LAMO without software automation. The main reason was the complicated HN calculation algorithm, which had been developed and used for several years. This algorithm was previously described in [6] and it is improved in this paper. The second reason is the future development of LAMO4, which contained $1201 \times 1901 \times 27=61643727$ spatial grid nodes. The number of lakes and rivers included in HN could be increased by changing the initial set of $\mathrm{HN}$ data, too. All these problems need an automated solution.

LAMO uses modelling environment GV [7]. The automation of this environment is done basically on the input/output data file level by creating the input data or by processing the data of results. The main initial data for the implementation of HN into a model are surfaces of layers and attaching data for rivers and lakes to the HM grid nodes set. In Section „Algorithms and Methods" some algorithms are described which are used in creation of $\mathrm{HN}$ and its implementation into the model. In Section „Architecture of Automation" software solution is described, which realizes these algorithms.

\section{ALGORITHMS AND METHODS}

\section{A. Creation of River Data}

The EMC software GDI [8] interpolates the model surface set. The data for rivers should be interpolated as a long line profile with the water level value by the program CRP [8] on each river profile line intersection point with cell edge of the model. The first stage of the LAMO development interpolation levels of long line profile of rivers was performed. The levels were based on the river level post data with the following correction with only on elevation lines based relief surface data. According to the algorithm, which is described in [6], the river long line should be below the relief surface level. The first calculations of the LAMO river flow balance confirmed the collecting of groundwater by rivers and also losing it, mostly in rivers. Source interpolated data for rivers were unreliable, because they were based only on the relief elevation data. The assumption was made that in those places the level of river should be below groundwater level of the Q2 aquifer:

$$
Z_{i}<=\operatorname{Head}_{Q 2}-L
$$

where $Z_{i}$ - level of the river for a grid node; Head ${ }_{Q 2}-$ groundwater level on the aquifer; $Q 2, L$ - constant value, which describes minimal difference between the river level and the $\mathrm{Head}_{Q 2}$. The decreasing level values on river long line profile must be supported when (1) is applied.

The problem of river water loss to an aquifer was solved by this correction. Obtaining of levels on river long line profile was realized as iterative process, because these levels were 
dependent on the groundwater levels formed in previous model simulation. The correction of the Gauja river long line profile is shown in Fig. 1. The main correction was realized near the river source that could be originated by the lack of the river level post data there and by the imperfection of the used linear interpolation algorithm. The river width value was computed by linear interpolation of the available river width data.

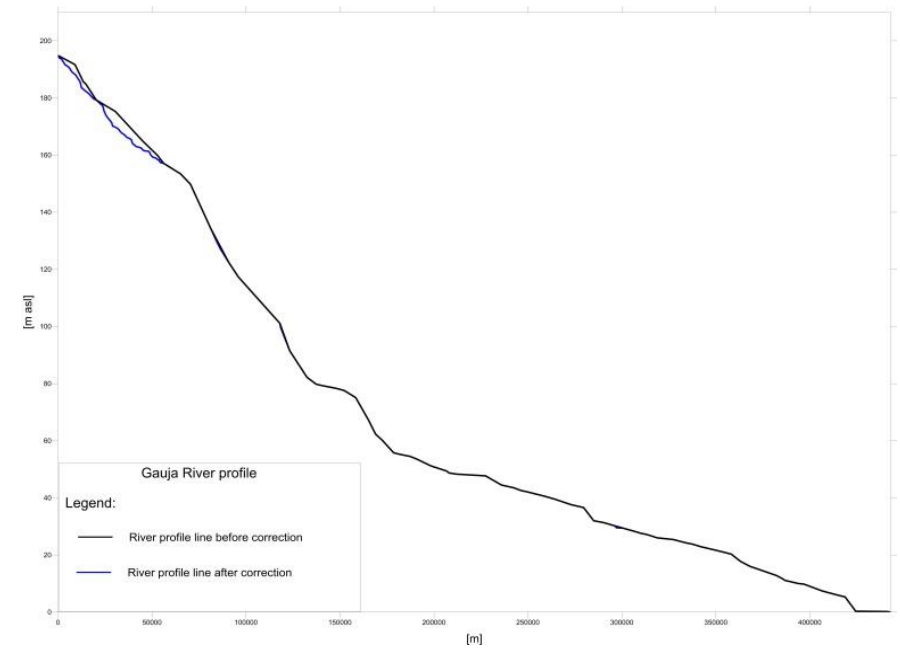

Fig. 1. Correction of levels on the Gauja river.

\section{B. Creation of River Attaching Data}

The rivers were incorporated into LAMO by attaching them to it. The attaching algorithm was performed by the GV Modflow module. The .RIV file (Fig. 2) shows how a river was attached to HM. The file had the address of the river attached node as row and column indexes. These addresses were converted to the model coordinates. The river was linked with the two nearest nodes and to the grid cell edge intersection point.



Fig. 2. Modflow River package file.

The conversion from the model cell address to the HM coordinates was given by the formula:

$$
\begin{aligned}
& x_{i}=(\text { column }-1) \times \text { step }+ \text { start } x \\
& y_{i}=\left(\text { rowcount }- \text { row }_{i}+1\right) \times \text { step }+ \text { starty }
\end{aligned}
$$

where $x_{i}, y_{i}$-coordinate of the node; column - column number; step - model plane approximation step; rowcount row count in model layer; startx, starty the coordinate of the left bottom corner of HM.
Some additional parameters are needed for the creation of river attaching data to $\mathrm{HM}$, such as the river width, attachment aquifer number and the coordinates of the two nearest model nodes to the intersection point.

The coordinates of the two nearest nodes can be calculated by the following formulas:

$$
\begin{aligned}
& x_{i}=\operatorname{INT}\left(\frac{x_{p}}{\text { step }}\right) \times \text { step } \\
& x_{i+1}=I N T\left(\frac{x_{p}}{\text { step }}+1\right) \times \text { step } \\
& y_{i}, y_{i+1}=y_{p}
\end{aligned}
$$

where $x_{p}$ and $y_{p}$ are the coordinates of the intersection point and step denotes the plane approximation step. These formulas are for the case when the intersection point is on the $\mathrm{Y}$ edge of the grid cell.

The attaching data file (Fig. 3) contains: coordinate $X$, coordinate $Y$, attaching layer, river id, river level and width, thickness of sediments and the river name. The attaching layer values are obtained by the algorithm described in Subsection "Immersion of Valleys of Rivers Into the HM Body".



Fig. 3. Example of river attaching file.

The lake attaching data file has a similar structure (Fig. 3) to the attaching data file of a river. The main difference is in the lake node set, because the river node set is based on a line, but the lake node set is based on an area. To include all nodes, which are located within the lake area, the blanking operation "Grid Blank" of the Surfer software is used. After the blanking every masked node value was changed to the $i d$ value of the lake.

In the case, when the lake area is narrow, this algorithm obtained only a few nodes (Fig. 4a). It was necessary to enlarge the number of nodes for narrow lakes.

To provide adequate number of the lake data, the described algorithm for lakes was merged with the algorithm for rivers, where the lake coast line was used as the river line. Then the nearest node outside the lake was added to the lake. In the Ciecere lake case (Fig. 4) the attaching node number increased from 10 to 40 .

The lake level was obtained from the LAMO relief with HN included. The calculation of the lake attaching layer was realized by the algorithm described in Subsection „Immersion of River Valleys Into the HM Body". The fragment of the lake attaching file is shown in Fig. 5. It contains $X$ and $Y$ coordinates of the lake node, attaching layer number, id, level, width, thickness of sediments and the lake name. 


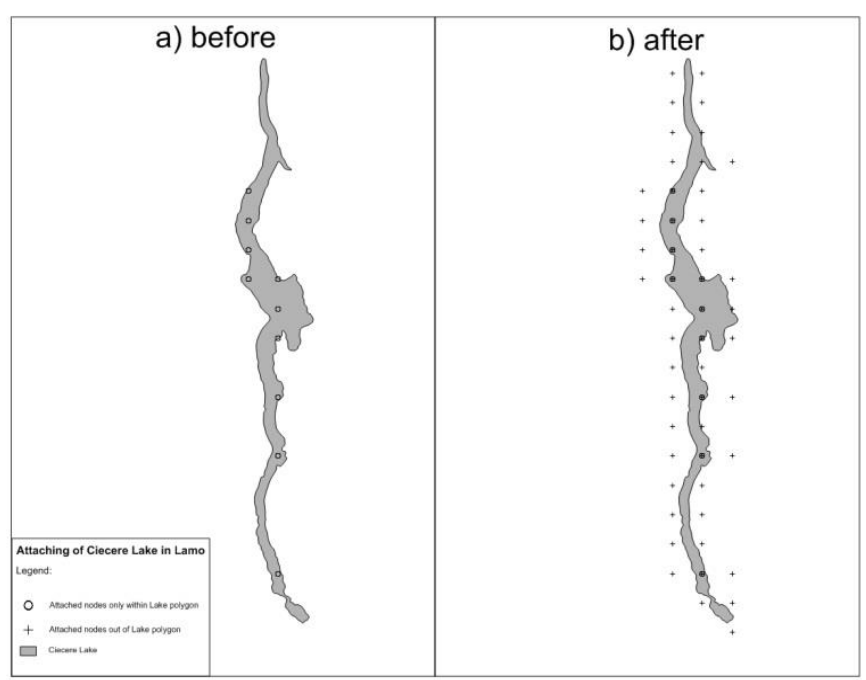

Fig. 4. Attaching of the Ciecere lake in LAMO.

\begin{tabular}{|c|c|c|c|c|c|c|}
\hline$\stackrel{0}{i}$ & 10 & & 20 & & 30 & 40. \\
\hline rd & Y_coord & Laye & er_Nr & Lak & e_i & d Relh_z \\
\hline $4 \overline{4} 3$ & $3 \overline{5} 4500$ & 31 & 0.7501 & 5 & & 1 Engure \\
\hline 444 & 354500 & 31 & 0.75 & 50 & 1 & Engures \\
\hline 444000 & 354000 & 31 & 0.75 & 50 & 1 & Engures \\
\hline 444500 & 354000 & 31 & 0.705 & 50 & 1 & Engures \\
\hline 5444000 & 353500 & 31 & 0.75 & 50 & & Engures \\
\hline
\end{tabular}

Fig. 5. Example of lake attaching file.

\section{Immersion of River Valleys Into the HM Body}

Including of $\mathrm{HN}$ data into the relief was done by the GDI interpolation software [8]. Sometimes the river valley cuts through geological layers. Then geological layers should be changed by accounting for presence of the valley. In the planes where the layer is cut, its thickness is set to 0.02 meter, but for aquifer $Q 2-1$ meter.

Surfaces of layers were used as the input data without accounting for valleys of rivers. Only for nodes of river attaching files data (Fig. 3) were processed to account for incised valleys. Data on all layers were compared by using the following formula:

$$
\text { Layer }_{i}<=\text { Layer }_{(i-1)} \text {-thickness_of_layer }
$$

where Layeri denotes the current layer node of the surface elevation and thickness_of_layer is the minimal thickness of the current layer.

All surfaces below the relief were changed and surfaces of layers were immersed below the incised part of the river by this algorithm. In the example (Fig. 6) of the cross section $2 W-2 E$, which intersects the Gauja river twice, the incisions of the river were not done. After applying the immersion algorithm, the full Gauja river valley was formed (Fig. 7).

\section{ARCHITECTURE OF AUTOMATION}

\section{A. Architecture}

Automation software for $\mathrm{HN}$ creation and incorporation into LAMO contains a set of programs and scripting files that are united in modules, which can be dependent from each other on the input/output data level. Basically algorithms are implemented in the Fortran programming language with some scripts written in the Surfer [9] BASIC and in the VBScript language. The scripting approach enables to use several algorithms for manipulations with surface data and for changing of the data format. The description of modules and names of main programs are given in Fig. 8.

Main programs:

1) CRP - processes data linear interpolation with the given plane approximation step;

2) GDI - interpolates prepared data as surface with the given plane approximation step;

3) izoliniju_vertibas_upei - adds new points for the river geometry with the same level as in point attribute data. The program allows adding of river level values only in decreasing order;

4) dig_dck_rez_to_bln - obtains river data correction points, where the river long line is above the surface level i.e., the relief surface level;

5) izoliniju_izveide - joins the geometry of isolines with their attribute - level;

6) grd_vistas_river_level_former - creates the river attaching data;

7) lamo_lakes_blank_2014 - creates the lake attaching data;

8) grd_on_river_correction - realizes incision of rivers on the geometry of HM;

9) grd_to_gwv_matrix - merges and changes the format of all layer surfaces to the GV Matrix file format.

Additional scripts for data type conversion, blanking and other mathematical operations on grid surfaces are used. The module control, data exchange and the union of modules is realized by MS-DOS BAT command scripts.

Unified approach used in batch files enables to change the plane approximation step and to use input data sets for HN. In the example (Fig. 9) names of input and output data files are declared as variables in rows 1-8, which are used as parameters for calling two other batch files in rows 9 and 11 . This fragment describes the $\mathrm{HN}$ incorporation into relief and representation of results as the Surfer map.



Fig. 9. Using parameters in the batch file. 
$2014 / 53$

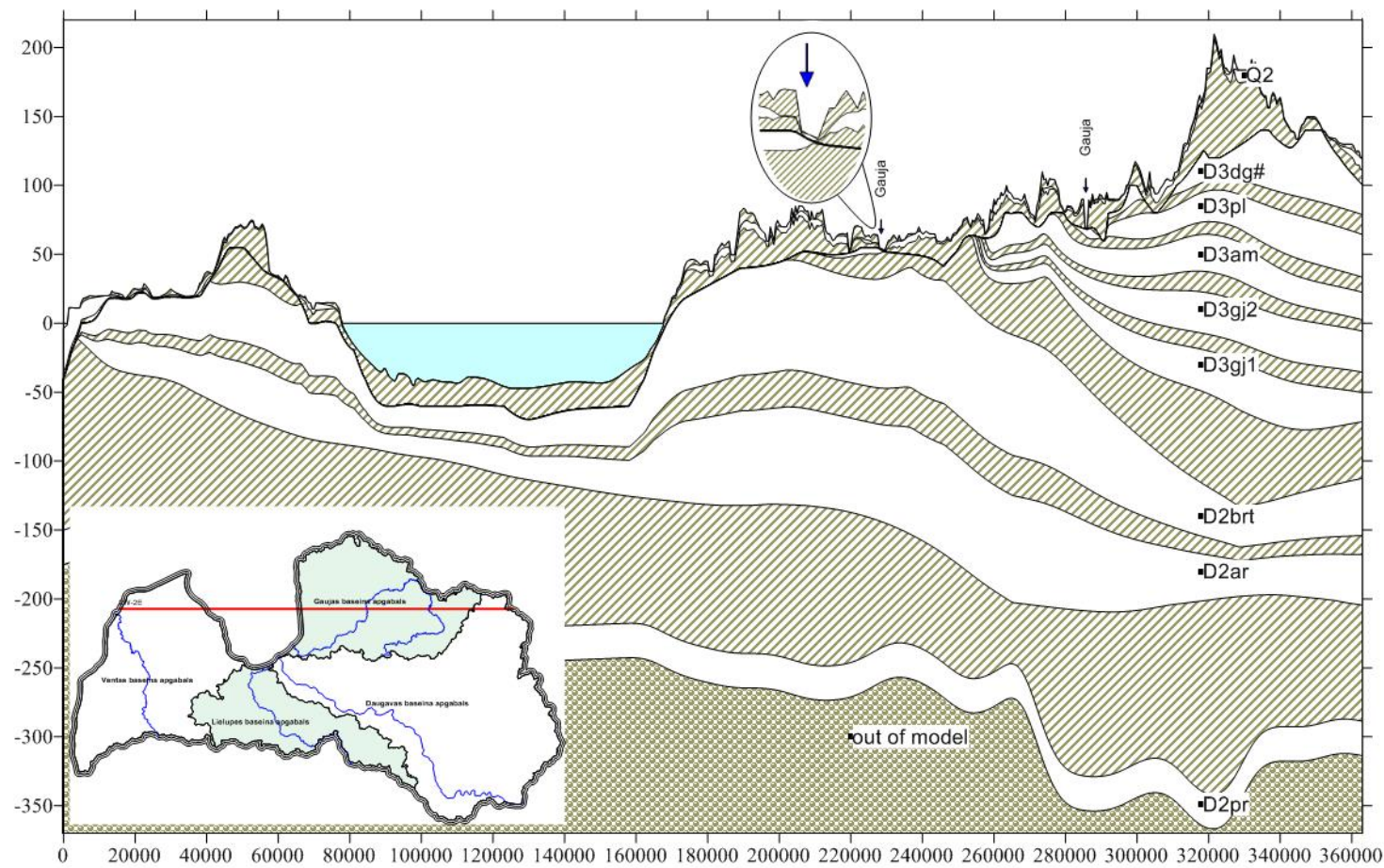

Fig. 6. Cross section $2 W-2 E$ before correcting the geometry.

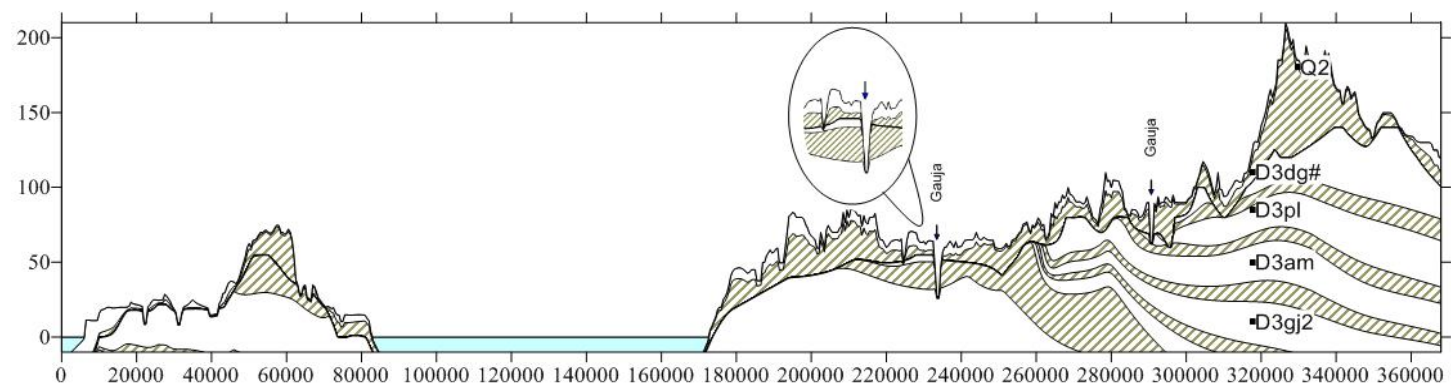

Fig. 7. Part of cross section $2 W-2 E$ after correcting the geometry.

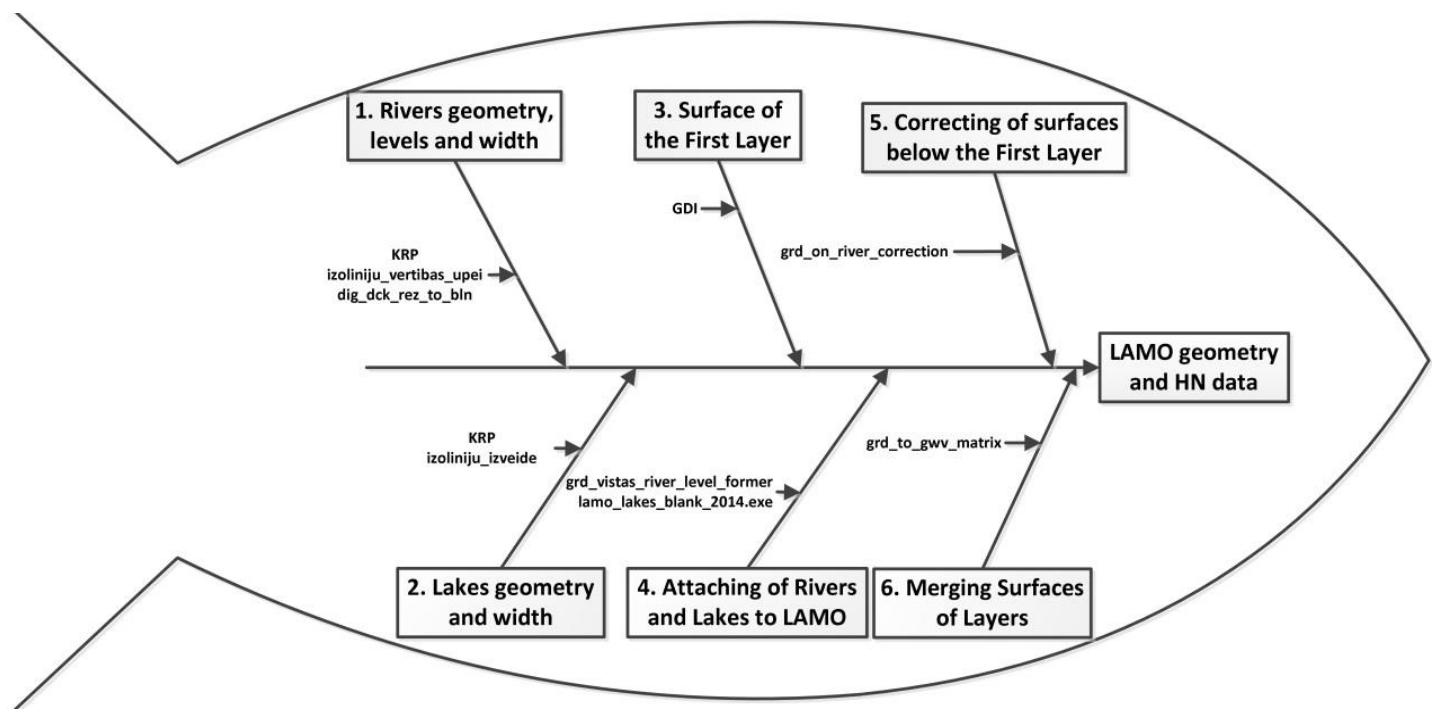

Fig. 8. Structure of important modules and programs for $\mathrm{HN}$ creation. 


\section{B. Performance}

This module set prepares LAMO3 geometry, HN attaching data and some other data for boundary conditions of HM in two hours. In the case of LAMO4 it will be approximately 8 hours.

Definitely, optimization of the software is possible - by minimizing conversion operations for data, by using data bases as storage, by combining more tasks under one, main program, by using parallelization approach for the software and by optimizing algorithms. The main advantages of the current solution are: the possibility of rapid change in every module and easy data control in each stage.

\section{CONCLUSION}

The described algorithms and methods were used in practice in creation of the LAMO data. The algorithms of river level calculation guarantee changing of values in decreasing way, integrity of level data between rivers of the same basin, between the river and the relief surface and between the river and groundwater head. Immersion of river valleys was realized by decreasing the level and thickness of LAMO surfaces in places, where the incision existed. Two algorithms were developed and implemented for attaching rivers and lakes to HM. Unifying of all LAMO surfaces in one file saved a lot of time when the HM geometry had to be changed.

The current software solution is very fragmentary and needs a lot of time to perform it. All modules can work uninterruptedly, without user's involvement. The prepared data can be checked in each stage on the file level. Parametric approach for the batch files enables to create HN data for different models with a different plane approximation step and different initial HN data without significant changes. This statement will be proved by the next model LAMO4, which will be with different plane approximation step $-250 \mathrm{~m}$. The main directions of the future development for this solution will be the improvement of algorithms and of software performance.

\section{ACKNOWLEDGEMENT}

In 2010-2012 the hydrogeological model of Latvia called LAMO was developed within the framework of the project "The Creating of Hydrogeological Model of Latvia to be used for Management of Groundwater Resources and for Evaluation of Their Recovery Measures". The project was cofinanced by the European Regional Development Fund.

\section{REFERENCES}

[1] A. Spalvins, J. Slangens, I. Lace, O. Aleksans, K. Krauklis, V. Skibelis, and I. Eglite, "Hydrogeological model of Latvia after increasing density of its hydrographical network," Scientific Journal of Riga Technical University, Boundary Field Problems and Computer Simulation, vol. 53, pp. 12-24, 2014

[2] Environment Modelling Centre, "Latvian Model," February 2013. [Online] Available: http://www.emc.rtu.lv/lamo.htm [Accessed Oct. 28, 2014].

[3] A. Spalvins, and U. Nulle. "Hydrogeological model for management and recovery of groundwater resources of Latvia," (in Latvian), Scientific Journal of Riga Technical University, Boundary Field Problems and Computer Simulation, vol. 49, pp. 7-13, 2011

[4] A. Spalvins, J. Slangens, and K. Krauklis, "Updating of Geological Data Interpolation Programs," Scientific Proceedings of Riga Technical University in series Computer Science, vol. 33, pp. 118-129, 2007.

[5] J. Slangens, and K. Krauklis, "Creating of Digital Relief Map for Regional Hydrogeological Model of Latvia," Scientific Journal of Riga Technical University, Boundary Field Problems and Computer Simulation, vol. 49, pp. 21-26, 2011.

[6] J. Slangens, K. Krauklis, and I. Eglite, "Incorporation of the Hydrographical Network into the Digital Map of the Ground Relief," Scientific Journal of Riga Technical University, Boundary Field Problems and Computer Simulation, vol. 45, pp. 45-52, 2010.

[7] Groundwater Modeling Software Specialists, "Environmental Simulations Incorporated," 2014. [Online]. Available: http://groundwatermodels.com, [Accessed Oct. 27, 2014].

[8] A. Spalvins, J. Slangens, R. Janbickis, and I. Lace, "Interpolation as an Important Tool for Creating Credible Hydrogeological Models," in Proc. of the 4th International Conference on Calibration and Reliability in Groundwater Modelling, ModelCARE'2002, 2002, pp. 84 - 87. [Online]. Available: http://www.emc.rtu.lv/PragaPoster2002.pdf, [Accessed Oct. 27, 2014].

[9] Golden Software Inc., "Surfer ${ }^{\circledR}$ 12," 2014. [Online]. Available: http://www.goldensoftware.com/products/surfer [Accessed Oct. 28, 2014].

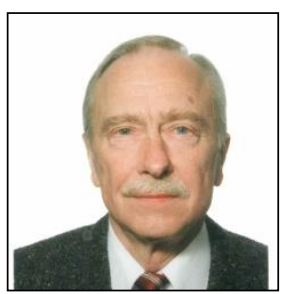

Janis Slangens was born in Latvia. In 1969, he graduated from Riga Polytechnical Institute (Riga Technical University since 1990) as a Computer Engineer. $\mathrm{He}$ is a Senior Researcher with the Environment Modeling Center. His research interests include computer modeling of groundwater flows.

E-mail: emc@cs.rtu.lv



Kaspars Krauklis received the Master's degree in Computer Systems from Riga Technical University in 2007 and the Certificate in Teaching of Engineering Sciences in 2005 from the Institute of Humanities, RTU. He is currently a Researcher with the Environment Modeling Center of Riga Technical University.

E-mail: emc@cs.rtu.lv 